approaches the characteristic fluorescence spectrum of methyl cholanthrene. The best sample indicates an identity between the fluorescence spectra of methyl cholanthrene and the cancerous fraction. The possibility that many forms of cancer may originate in the metabolic production of methyl cholanthrene or related substances from the bile acids, or perhaps from the sterol or sex hormones of the body, has been mentioned elsewhere. The spectrographic work has been done with the aid of Dr. S. M. Rubens and Professor J. Kaplan of the University of California at Los Angeles.

\section{The Low Frequency Fundamental Band of Ethane at $12 \mu$}

RALPH G. OWens* and E. F. Barker

Harrison M. Randall Laboralory of Physics, University of Michigan, Ann Arbor, Michigan

December 29, 1941

$I^{\mathrm{N}}$

$\mathrm{N}$ preparing for a study, now under way, of the infra-red spectra of deuterium bearing ethanes we have reexamined the low frequency fundamental absorption band of $\mathrm{C}_{2} \mathrm{H}_{6}$. This band was first resolved by Levin and Meyer, ${ }^{1}$ with a spectrometer somewhat less effective than the best now available. They showed that it is of the degenerate perpendicular type, consisting of a family of $Q$ branches spaced at intervals of approximately $2.6 \mathrm{~cm}^{-1}$. The significance of this and other details of the ethane spectrum has been discussed more recently by Crawford, Avery, and Linnett. ${ }^{2}$

The pattern of this band as obtained with our present instrument is shown in Fig. 1, and the wave numbers

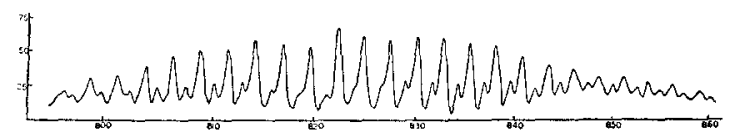

FIG. 1. The $12 \mu$ band of ethane; percent absorption $v s$, wave number.

(reduced to vacuum) for the various maxima are listed in Table 1. These measurements reveal two features worthy of remark. (1) The spin effect, which slightly accentuates every third line, is clearly revealed. Since the band center must lie one-half interval toward lower frequencies from the strongest line, its position may be definitely fixed. We

TABLE I. Observed line positions in wave numbers reduced to vacuum.

\begin{tabular}{cccc}
\hline & & & \\
\hline & Minor peaks & Major peaks & Minor peaks \\
\hline $\mathbf{8 5 9 . 5 4}$ & $\mathbf{8 5 7 . 7 2}$ & $\mathbf{8 2 2 . 2 0}$ & \\
$\mathbf{8 5 6 . 7 0}$ & $\mathbf{8 5 5 . 2 3}$ & $\mathbf{8 1 9 . 5 2}$ & \\
$\mathbf{8 5 3 . 9 5}$ & $\mathbf{8 5 2 . 7 6}$ & $\mathbf{8 1 6 . 7 9}$ & 815.78 \\
$\mathbf{8 5 1 . 3 4}$ & $\mathbf{8 5 0 . 0 8}$ & $\mathbf{8 1 4 . 2 2}$ & 812.96 \\
$\mathbf{8 4 8 . 5 9}$ & $\mathbf{8 4 7 . 5 8}$ & $\mathbf{8 1 1 . 0 0}$ & 810.35 \\
$\mathbf{8 4 6 . 0 6}$ & $\mathbf{8 4 4 . 6 0}$ & $\mathbf{8 0 8 . 9 4}$ & 807.60 \\
$\mathbf{8 4 3 . 3 6}$ & $\mathbf{8 4 2 . 0 6}$ & $\mathbf{8 0 6 . 3 9}$ & $\mathbf{8 0 5 . 0 0}$ \\
$\mathbf{8 4 0 . 7 5}$ & $\mathbf{8 3 9 . 5 0}$ & $\mathbf{8 0 3 . 8 4}$ & $\mathbf{8 0 2 . 3 6}$ \\
$\mathbf{8 3 8 . 0 8}$ & $\mathbf{8 3 6 . 8 1}$ & $\mathbf{8 0 1 . 2 4}$ & 798.98 \\
$\mathbf{8 3 5 . 3 9}$ & $\mathbf{8 3 4 . 1 2}$ & 798.69 & 797.18 \\
$\mathbf{8 3 2 . 6 7}$ & $\mathbf{8 3 1 . 5 3}$ & 796.39 & \\
$\mathbf{8 3 0 . 0 5}$ & $\mathbf{8 2 9 . 0 2}$ & & \\
$\mathbf{8 2 7 . 4 0}$ & & & \\
$\mathbf{8 2 4 . 7 6}$ & & & \\
\hline
\end{tabular}

obtain for it the value $820.82 \mathrm{~cm}^{-1}$. The high frequency side of the band is the more intense because of lower initial $K$ values. (2) Approximately midway between the major peaks a series of minor absorption maxima are observed. These appear to be relatively stronger near both limits of the band, and less intense in the neighborhood of the center, suggesting two subsidiary transitions. They probably arise from the change $1 \rightarrow 2$ in the same vibrational quantum number for which the transition $0 \rightarrow 1$ yields the principal band. The first excited state involves an internal angular momentum which, for the upper transitions, may either increase or decrease, with resulting bands at frequencies above and below that of the fundamental. From the present measurements we are not able to determine the centers for these low intensity bands.

* Now at Colorado School of Mines.

1 A. Levin and C. F. Meyer, J. Opt. Soc. Am. and Rev. Sci. Inst. 16 137 (1928).

2 B. L. Crawford, W. H. Avery and J. W. Linnett, J. Chem. Phys. 6 682 (1938).

\section{Erratum: Chain Length and Chain-Ending Processes in Acetaldehyde Decomposition}

\author{
(J. Chem. Phys. 7, 1080 (1939))
}

Milton Burton, H. Austin Taylor and Thomas W. Davis New York University, University Heights, New York, New York January 3, 1942

In a paper under the above title, ${ }^{1}$ the chain lengths in the azomethane-induced decomposition and in the photolysis of acetaldehyde at equal rates of free radical production and equal aldehyde concentrations were calculated from the available data. It has been called to our attention by Professor $O$. K. Rice that the calculation for the induced decomposition is in error. The chain length per free radical should have the value $\sim 220$. Comparison of this value with that for the photolysis given by us as 844 vitiates our conclusions drawn in the paper cited relative to the chain-ending processes and would necessitate a new analysis of the possible reactions involved. It is our understanding that Professor Rice plans to discuss such possibilities in a forthcoming paper.

1.T. Chem. Phys. 7, 1080 (1939).

\section{Erratum: Ethylene-Ethane and Propylene- Propane Equilibria}

(J. Chem. Phys. 10, 78 (1942))

G. B. Kistiakowsky AND A. Gordon NiCKLE Department of Chemistry, Harvard University,
Cambridge, Massachusetts

$T$ ROUGH an error Dr. Nickle's name was omitted from this Letter to the Editor. The authors should have been listed as above. 